\title{
DETECTING A NON-GAUSSIAN STOCHASTIC BACKGROUND OF GRAVITATIONAL RADIATION
}

\author{
STEVE DRASCO \& ÉANNA É. FLANAGAN \\ Newman Laboratory of Nuclear Studies \\ Cornell University, Ithaca, New York 14853-5001, USA
}

\begin{abstract}
We derive a detection method for a stochastic background of gravitational waves produced by events where the ratio of the average time between events to the average duration of an event is large. Such a signal would sound something like popcorn popping. Our derivation is based on the somewhat unrealistic assumption that the duration of an event is smaller than the detector time resolution.
\end{abstract}

Consider a large collection of similar gravitational wave sources. If we cannot resolve the individual signals produced by these sources and know only their statistical properties, the signals form a stochastic background. We group stochastic backgrounds into the following two classes: those for which the individual sources have (i) average redshift $z \gg 1$ and (ii) average redshift $z \sim 1$. Typically in case (i) the number of individual sources is large enough that the duration of events is long compared to the time between events, and then the central limit theorem enforces Gaussianity of the background. However, for some sources in case (ii) the anticipated number of individual sources might be small enough that this argument breaks down and a non-Gaussian stochastic background might be expected. Examples of (ii) are supernovaet, binary inspirals, and young neutron stars. Well developed data analysis methods exist for detecting Gaussian backgrounds. Here we derive a data analysis method for a non-Gaussian background under idealized assumptions; further work is needed to obtain realistic detection methods.

Assume a pair of co-located, aligned detectors with outputs $h_{i}^{k}=n_{i}^{k}+s^{k}(i=$ 1,2) of uncorrelated Gaussian noise $n_{i}^{k}$ and possibly a stochastic background signal $s^{k}$. Here $i$ labels the detectors and $k$ with $1 \leq k \leq N$ labels the time samples of the detector output. We assume the following probability distribution for the stochastic background signal:

$$
p(\mathbf{s} \mid \xi, \alpha)=\prod_{k}\left\{\frac{\xi}{\sqrt{2 \pi \alpha}} \exp \left[-\frac{\left(s^{k}\right)^{2}}{2 \alpha}\right]+(1-\xi) \delta\left(s^{k}\right)\right\},
$$

where $\mathbf{s}=\left(s^{1}, \ldots, s^{N}\right)$. Thus, the individual time samples are assumed to be statistically independent and identically distributed; this is because we assume the duration of events is smaller than the detector sampling time, so each event affects only one time sample ${ }^{a}$. In Eq. (11), the parameter $\xi$ is the probability that a randomly-chosen data sample will contain an event, and $\alpha$ is the signal strength. The Gaussian case is recovered in the limit $\xi \rightarrow 1$.

We use Bayesian methods to motivate the choice of detection statistic. Let $P^{(1)}$ be the probability that a signal is present in the data (posterior) and $P^{(0)}$ be the probability that a signal will be present (prior). The relation between these

${ }^{a}$ Detector time resolutions can be on the order of milliseconds and even supernovae waveforms can be on the order of hundreds of milliseconds, so this assumption is somewhat unrealistic.

proceedings: submitted to World Scientific on November 3, 2018 
probabilities is given in terms of the likelihood ratio $\Lambda$ by

$$
\frac{P^{(1)}}{1-P^{(1)}}=\Lambda \frac{P^{(0)}}{1-P^{(0)}} .
$$

A natural detection criterion is to threshold on $P^{(1)}$, which is equivalent to thresholding on $\Lambda$. Thus, we will say that the signal has been detected if $\Lambda>\Lambda_{*}$ for some threshold $\Lambda_{*}$ 用.

To compute $\Lambda$ we need only detector data and the probability density functions for the signal and for the noise in each detector. We find

$$
\Lambda=\int d^{N} s \frac{p_{\mathcal{N}_{1}, \mathcal{N}_{2}}\left(\mathbf{h}_{1}-\mathbf{s}, \mathbf{h}_{2}-\mathbf{s}\right)}{p_{\mathcal{N}_{1}, \mathcal{N}_{2}}\left(\mathbf{h}_{1}, \mathbf{h}_{2}\right)} \tilde{p}_{\mathcal{S}}^{(0)}(\mathbf{s}),
$$

where $\mathbf{h}_{i}$ is the vector $\left(h_{i}^{1}, \ldots, h_{i}^{N}\right), p_{\mathcal{N}_{1}, \mathcal{N}_{2}}\left(\mathbf{n}_{1}, \mathbf{n}_{2}\right)$ is the joint probability density of the detector noises $\mathbf{n}_{1}$ and $\mathbf{n}_{2}$, and $\tilde{p}_{\mathcal{S}}^{(0)}(\mathbf{s})$ is the probability density that the signal $\mathbf{s}$ is present in the data, given that some signal is in the data. We take $\tilde{p}_{\mathcal{S}}^{(0)}(\mathbf{s})=$ $\int d \xi \int d \alpha p(\mathbf{s} \mid \xi, \alpha) \tilde{p}^{(0)}(\xi, \alpha)$, where $\tilde{p}^{(0)}(\xi, \alpha)$ is the prior distribution for the signal parameters $\xi$ and $\alpha$. Combining these equations and assuming uncorrelated white Gaussian noise of unit variance in each detector 1 we obtain

$$
\Lambda=\int d \xi \int d \alpha \Lambda(\xi, \alpha) \tilde{p}^{(0)}(\xi, \alpha)
$$

where

$$
\ln \Lambda(\xi, \alpha)=\sum_{j} \ln \left(1+\xi v^{j}\right)
$$

and

$$
v^{j} \equiv(1+2 \alpha)^{-1 / 2} \exp \left[\left(h_{1}^{j}+h_{2}^{j}\right)^{2} \frac{\alpha}{4 \alpha+2}\right]-1 .
$$

The detection statistic we suggest is the maximum likelihood statistic

$$
\Lambda_{\max }=\max _{\alpha, \xi} \Lambda(\xi, \alpha),
$$

which we expect to be large when $\Lambda$ itself is large. The maximization can be carried out numerically ${ }^{t}$. If a signal is detected, then the values $\xi_{\max }$ and $\alpha_{\max }$ which achieve the maximum give estimators of the true values of $\alpha$ and $\xi$. One can also form a posterior distribution for $\alpha$ and $\xi$ from the function $\Lambda(\alpha, \xi)$ and thereby attempt to distinguish between Gaussian and non-Gaussian backgrounds.

\section{References}

1. V Ferrari et al, Gravitational wave background from a cosmological population of core-collapse supernovae, (Mon. Not. Roy. Astron. Soc. 303, 247, 1999)

\footnotetext{
${ }^{b}$ We restrict attention here to finding a useful statistic and ignore the issue of how to choose the value of the threshold, which may be done using either Bayesian or frequentist methods.

${ }^{c}$ We expect to be able to relax this assumption in the near future.

${ }^{d}$ While such a search is computationally intensive, we have had some success with numerical simulations using small (length $\sim 10^{6}$ ) data segments.
}

proceedings: submitted to World Scientific on November 3, 2018 